\title{
Multiple Wechselwirkungen
}

Diabetes mellitus und Parodontitis beeinflussen sich gegenseitig über systemische Entzün-

dungsprozesse und Insulinresistenz. Diabetiker sollten daher regelmäßig auf Parodontitis untersucht werden, zumal deren erfolgreiche Therapie auch die glykämische Kontrolle verbessert.

Im Mund kommen bis zu tausend verschiedene Keimarten vor. Beim Gingiva-Gesunden überwiegen anaerobe Stäbchen und Kokken. Bei Parodontitis ist die Flora durch das Überwiegen gramnegativer anaerober Stäbchen charakterisiert. In Experimenten konnte gezeigt werden, dass bei fehlender Mundhygiene die Akkumulation bestimmter Mikroorganismen innerhalb von 2-3 Wochen zu Entzündungen an der Gingiva führt. „Der bakterielle Biofilm ist der Ausgangspunkt“, sagte Prof. Jörg Meyle, Ärztlicher Direktor des Zentrums für Zahn-, MundKieferheilkunde der Universität Gießen. Die Parodontitis führe zur Beeinträchtigung der Kaufunktion und zum Zahnverlust mit sich daraus ergebenden Veränderungen der Ernährung.

Bei der zunächst lokalen Entzündungsreaktion werden vermehrt proinflammatorische Zytokine wie Interleukin-1, -6, TNF-alfa, PgE freigesetzt, die dann in die Blutbahn gelangen und eine systemische Inflammation induzieren. „Diese führt zu einer Zunahme der Insulinresistenz und zu Typ-2-Diabetes“, so Meyle. Durch Hyperglykämie entstehen vermehrt Advance Glycation Endproducts (AGEs), die die Entzündung verstärken.

Diabetes mellitus ist ein Risikofaktor für die Parodontitis Untersuchungen ergaben, dass bei Diabetikern ein hyperinflammatorischer Monozyten-Phänotyp exsistiert, der erhöhte lokale proinflammatorische Zytokin-Spiegel erzeugt. Zudem induziert eine chronische Hyperglykämie einen proinflammatorischen Zustand in der gingivalen Mikrozirkulation mit erhöhter vaskuläre Permeabilität. Auch werden Leukozyten und Endothelzellen aktiviert. „Die leukozytenvermittelte mikrovaskuläre Schädigung trägt wesentlich zur parodontalen Gewebedestruktion bei Diabetikern bei“, so Meyle. Und man habe eine erhöhte Immunreaktivität für AGEs in der Gingiva von Diabetikern mit Parodontitis im Vergleich zu Nichtdiabetikern nachweisen können. Die Lokalkonzentration von Markern für den oxidativen Stress sind erhöht. „AGEs in der Gingiva von Diabetikern sind Ausdruck eines gesteigerten Stresses und ein potenzieller Mechanismus für die beschleunigte Gewebedestruktion.“

\section{Bindeglied ist die Insulinresistenz}

Schon Übergewicht ist ein Risiko für Parodontitis, und zwar unabhängig von Alter, Geschlecht, Rasse und Rauchen. „Das Bindeglied dürfte die Insulinresistenz sein“, so Meyle. Insgesamt hätten Diabetiker bei einer schlechten glykämischen Kontrolle ein etwa dreifach höheres Risiko für Parodontitis. Der Zusammenhang zwischen Diabetes und Parodontitis ist bidirektional, d.h. sie begünstigen sich gegenseitig. Hier stellt sich die Frage, ob durch eine effektive Parodontitistherapie die Stoffwechseleinstellung verbessert werden kann. „In einer Metaanalyse konnte dies weniger für den Typ-1-, jedoch für den Typ-2-Diabetes nachgewiesen werden“, so Meyle. Bei Typ-2-Diabetikern konnte der $\mathrm{HbA}_{1 \mathrm{c}}$-Wert um 0,57\%-Punkte gesenkt werden, und zwar unabhängig von der antidiabetischen Therapie. Auch eine Antibiotikagabe senkte den $\mathrm{HbA}_{1 \mathrm{c}}$-Wert um 0,27\%-Punkte. „Eine Parodontitistherapie kann zur Verbesserung der glykämischen Kontrolle beitragen“, so Meyle. Zahnärzte sollten daher bei Parodontitispatienten die metabolische Kontrolle abfragen.

Gute Glukoseeinstellung verbessert die Zahngesundheit „Nicht nur mikro- und makrovaskuläre, sondern auch die oralen Komplikationen wie Gingivitis und Parodontitis gilt es zu verhindern“, sagte Prof. Petra-Maria Schumm-Draeger, vom Klinikum München-Bogenhausen. Gefragt sei ein engagiertes proaktives Diabetesmanagement. Die Mundgesundheit sei für die Lebensqualität und die Prognose bei Diabetes wichtig, da die systemische Entzündung Gefäßkomplikationen induzieren könne. „Die Parodontitis wird aber von vielen Diabetikern nicht wahrgenommen, da sie keine Schmerzen verursacht", so SchummDraeger. Folge sei eine verzögerte Diagnose und Behandlung.

Dass durch eine frühzeitige intensive glukosesenkende Therapie die vaskuläre Komplikationsrate und die Gesamtmortalität günstig beeinflusst werden kann, zeigen die Ergebnisse der UKPD-Studie. „Der signifikante Unterschied zwischen den konventionell und intensiv behandelten Diabetikern zeigte sich auch im Verlauf nach Ende der Studie“, so Schumm-Draeger. 10 Jahre danach lag die Inzidenz einer mikroangiopathischen Komplikation in der primär intensiv behandelten PatientenGruppe um 24\%, die Rate an Myokardinfarkten um 15\% und die Gesamtmortalität um 13\% niedriger. Auch in der Steno-2Studie konnte durch eine intensivierte Antidiabetikatherapie die kardiovaskuläre Ereignisrate um 55\% reduziert werden.

Kooperation zwischen Diabetologe und Parodontologe Zwischen Parodontitis und Hyperglykämie bestehen viele Wechselwirkungen. So sei das Risiko eines tödlichen kardiovaskulären Ereignisses bei fortgeschrittener Parodontitis um das 2,3-Fache und das Risiko für ein tödliches renales Ereignis sogar um das 8,5-Fache erhöht, so Schumm-Draeger. Angesichts der Wechselwirkungen ist ein ganzheitlicher interdisziplinärer Ansatz nötig. „Die Mundgesundheit muss in das Diabetesmanagement einbezogen werden“, so Schumm-Draeger. Der Zahnstatus müsse erhoben und routinemäßig kontrolliert werden, am sinnvollsten mit einem standardisierten Fragebogen. Bei schlechtem Zahnstatus und CRP-Erhöhung müsse eine zeitnahe Überweisung zum Zahnarzt erfolgen. Auch sollte das zahnärztliche Team an den Diabetes denken und eventuell mal ein Blutdruck-Screening machen.

Dr. med. Peter Stiefelhagen

Quelle: Kongresses „Innere Medizin fachübergreifend - Diabetologie grenzenlos" 01.03.2013 in München. 